\title{
Research on the development of Chinese traffic cloud computing
}

\author{
Chen Hua \\ School of Business, University of Beifang nationalities, Yinchuan Ningxia, China \\ hua_0110@163.com
}

Keywords: cloud computing; traffic cloud computing; intelligent transportation; transportation system

\begin{abstract}
Traffic is an important area of cloud computing application and development, and the traffic cloud computing is a key means to provide powerful support for the basic service and promote the coordinated development of the transport system by service model innovation and information resources integration of cloud computing. In this paper, the traffic cloud computing organization figure is provided, the current situation and problems of Chinese traffic cloud computing development are detailed analyzed, meanwhile, for such specific issues, the corresponding development strategies are given. We hope to provide a reference for coordinated development of Chinese transportation systems, as well as the practice applications of cloud computing in the transport area.
\end{abstract}

\section{Introduction}

As the popularity of personal computers and the Internet in the last century, modern Internet derived technologies just like cloud computing is growing so crazy as an IT revolution, and showed a very broad development prospects. In recent years, global cloud computing market is growing rapidly, many countries or regions with powerful information and industries have given great attention to the cloud computing, and most of them have taken the cloud computing as the focus of future industry development strategy. They research and publish the industry development strategic planning, and try to accelerate the deployment of cloud computing infrastructure and platform construction also with its applications.

In China, the government attaches great importance to the development of the cloud computing, and put it as an important strategic emerging industry direction to support during "the Twelfth Five Plan" [1]. Meanwhile, in order to expedite the development and implementation of cloud computing, The CPC Central Committee and State Council, Ministry of Industry and Information Technology, Ministry of Finance, National Development and Reform Commission have issued "The CPC Central Committee and State Council's Decision on accelerating the cultivation and development of strategic emerging industries"[2], "Notice on cloud computing service innovation and development of pilot and demonstration work "[3], "guiding opinions on Accelerating the development of hightech service industry"[4] and "categorized list of government procurement Categories"[5] one after another. In these official documents, cloud computing service innovation, application demonstration and promotion are all proposed to accelerate.

January 30, 2015, The CPC Central Committee and State Council issued the "opinions on promoting the cloud computing innovation and fostering the new format of information industry" [6], which proposed: "By 2017, cloud computing applications will be deepened in key areas, the industrial chain will be integrate and clear basically, the development pattern with powerful security, collaborative promotion of service innovation, technological innovation and management innovation will be formed preliminarily, and bring related industries to develop rapidly. Service capabilities will be significantly heightened; innovation capability will be enhanced; application demonstration will be remarkably effective; infrastructure will be gradually optimized; and the security will be integrated. By 2020, the cloud computing application will be popular; service capabilities will come up to international advanced level, the key technology of cloud computing will be mastered; a number of enterprises with strong international competitiveness will appear. " 
The transportation is one important area of cloud computing application. The traffic cloud computing is defined as an information service innovation model with traffic resource sharing, which provides an advanced, integrated, safe, automated, expandable and open platform or traffic systems by cloud computing. The traffic cloud computing is a main body of the cloud computing services innovation and development.

However, with the rapid development of Chinese national economy in recent years, various cars and vehicles have risen so rapidly, traffic flow have also grown along with it, and the traffic congestion and so lead to energy issues, social issues and environmental issues have become more and more severe, and it make the importance of traffic programming, building and coordination management prominent. The Chinese government has a deep understanding about it. The Ministry of Transport issued "transportation deepen reform opinions" [7], which states that: "Explore the management model with planning, construction and operation integrated. Improve the transportation technology innovation mechanism; strengthen the major scientific attack and technological achievements transformation; promote the technology of Next Generation Internet, The Internet of Things and Big Data's application in traffic area.” Therefore, how to use a new generation of information technologies to promote the healthy development of transport system has become particularly urgent.

In this paper, the structure of traffic cloud computing platform, the application status and characteristics of cloud computing in Chinese transportation, the problems of traffic cloud computing development and the strategies have researched. We hope it can provide a reference to cloud computing application in traffic area.

\section{Traffic Cloud Computing Platform Organization Structure}

Traffic cloud computing can use data storage, unified resource management and information security technology to solve a large number of problems effectively like information scattered, huge amounts of data cannot be integrated, a large number of manpower, material and financial resources are wasted etc.[8]. Meanwhile, traffic cloud computing can bring about scale effect, open effect and innovation effect [9]. It also means that the cloud computing will break the special high cost communication and publication system, and more users can join in by hiring the services.

From the current trend of applications, traffic cloud computing has the same service type and service model with the other industry cloud computing. That is, it includes the public cloud, private cloud and hybrid cloud for service type, and it includes the Infrastructure as a Service (IaaS), Software as a Service (SaaS) and Platform as a Service (PaaS) according to the service model [10]. However, in the traffic cloud computing platform, the cloud resource service based on data center is the core part of the previous system, and the organizational structure shown in Figure 1:

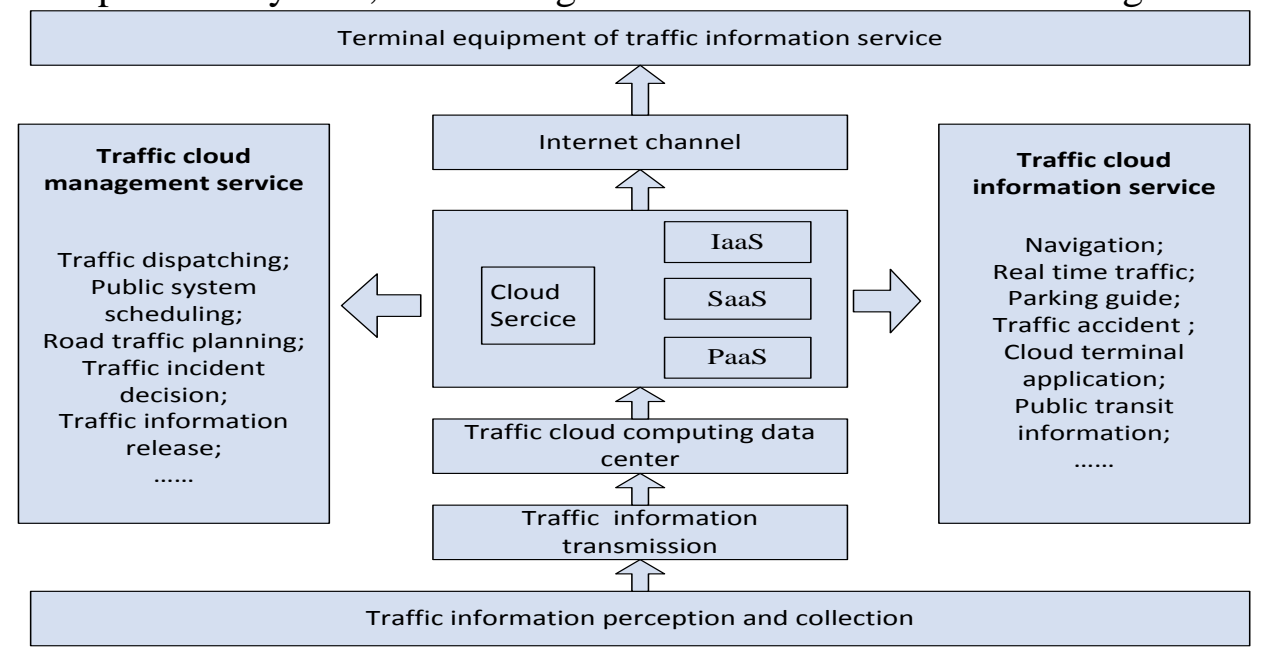

Figure 1. The traffic cloud computing platform organizational structure. 


\section{The Status and Existing Problems of Chinese Traffic Cloud Computing Development}

The Chinese government attaches great importance to the development of traffic cloud computing, the platforms are being constructed, and the specific application exploration is also being accelerated.

With the rapid development of new technology fields like the Internet, The 4G, The Internet of Things, Big Data etc., the IT services is constantly changing the traditional service model, and is using to make promotion of modern management. The Chinese government attaches great importance to the new technologies innovative and the development of service models, six cities have been established as pilot to promote the application and development of cloud computing (In October 2010, Ministry of Industry and Information Technology, National Development and Reform Commission have jointly issued "Notice on cloud computing service innovation and development of pilot and demonstration work", and have established Beijing, Shanghai, Shenzhen, Hangzhou, Wuxi as five advance pilot and demonstration cities to carry out cloud computing service innovation and development. In January 2014, Ministry of Industry and Information Technology, National Development and Reform Commission have jointly issued Harbin as a pilot city again.). However, the implementation of such decision is late in 2010, there is a substantial exploration of industry cloud computing with the government support and some enterprises attempting, and so have many traffic cloud computing platform been built after 2013. The platform is now has a more clear organizational structure, and the form of service has being more and more plentiful, but it is in building period, the content of service is still limited, and the specific applications are still in exploration.

The technology of traffic cloud computing can support the basic applications, but the core technology has yet to wait for breakthrough.

The cloud computing was originally generated by a service model innovation, and all the relevant technology was evolving with needs improvement which is based on the original service application model. From the development status of Chinese cloud computing and the preliminary application in traffic area, the basic techniques can still be met. One of the most basic virtualization technology had appeared in IBM's mainframe systems as early as the 1960s, it is gradually mature and its application in international commercial field is also rising by multi-core systems, clusters, distributed systems and grid having widely deployed. Server virtualization, desktop virtualization, application virtualization, platform virtualization, storage virtualization etc., these technologies have been used in traffic cloud computing at present. However, some of the key technologies and their innovative application in China are still have to face some challenges. For example, data privacy, unpredictable performance, data transmission bottlenecks, scalable storage capacity and other special technical bottlenecks under cloud computing model; as well as the system software, platform software and database management systems etc. specific weaknesses in China.

In summary, the Chinese government pays more attention to the development of traffic cloud computing, the application and technology have being deepened, but there are still such problems in general.

\subsection{Lack of overall planning for the whole layout}

Traffic cloud computing is embedded in all segments of traffic industry chain, which involves hardware manufacturers, software developers, systems integrators, telecommunication operators, internet service providers and so on. The development of traffic cloud computing is depend on the promotion of all segments and all the types of businesses together. However, in China, the development of most industry cloud computing has been promoted by the ways of pilot, special project, demonstration etc., there is no more system planning and overall layout from the national macro level to think about the relationship between cloud applications and their roles in industry chain. So the local governments also tend to gain pilot, special project and demonstration, pursue the hot area, invest so much money, but the local, repetitive construction is very easy appeared for lack understanding of the entire industry, not in-depth research on the application requirements and not clear development expectation. The traffic cloud computing companies are trying to build their 
own cloud services, and there is no more close technical cooperation and clear division of work, so much problems like lower service scale, small investment and high repetition rate, blind service spots and other issues have being brought about.

\subsection{Safety and trust system have not been established}

Security, stability and quality of internet service are the three important factors need to be first considered for traffic cloud computing. Ministry of Industry and Information Technology Telecommunications Research Institute 2013 survey data showed that the Chinese cloud computing users are most concerned with the stability, security and network quality of cloud services, and the cloud service price, satisfaction of customer service, types of cloud services, brand popularity are the next. It is not hard to understand from the safety and trust system of present Chinese industry cloud computing application services. So far, in China, there are only laws and regulations of behavior management for personal information collection, use and processing, and there are rarely the other laws and regulations of information security and data protection. The legal system is not comprehensive at all.

\subsection{There are some difficulties to promote the application}

The cloud computing has provided the technical support for information sharing and business collaboration. The development of transportation needs cloud computing service model to promote the integration of information resources and innovation of management system. But, in Chinese transportation department, cloud service is just put into the procurement catalog; it is lack of the feasible standards, strict procurement control, objective assessment and timely afterwards management, so the further promotion of traffic cloud application is limited. In addition, purchasing outsourcing practices are not widely accepted in all levels of Chinese information construction, too much self- construction lead to the duplication and resource wasting. Their own business data to be generated in the self- construction system are unwilling to open use within the industry for data mining and application. Some advanced information technology departments take a wait-and-see attitude to the cloud computing promotion and application because their temporary business needs can be satisfied, so they did not play a leading role in the development of it. All of these factors brought some obstacles to the traffic cloud computing development and application.

\section{Development Strategies of Chinese traffic cloud computing}

\subsection{The government should strengthen the overall planning, and to promote industrial resource integration.}

The CPC Central Committee and State Council, Ministry of Communications, Ministry of Industry and Information Technology, Ministry of Finance, National Development and Reform Commission should work together to strengthen the overall planning after fully investigating the existing cloud platforms and their service status quo. Furthermore, they should give guidelines to the industry chain healthy and stable development, and for the enterprises of hardware manufacturers, software developers, system integrators, telecommunications operators and Internet service provider etc. cooperative development. Meanwhile, local government should be encouraged to participate the traffic cloud computing construction, and make them play a guiding role to strengthen the construction of unified standards and improve traffic cloud platform integrated services capabilities.

\subsection{Construct and improve the legal system to ensure the safety of industrial applications}

The departments of government and industry supervisor should investigate the development and application status of traffic cloud computing, formulate relevant supervisor policy and make the power and obligation relationship of users and service providers clear, remove the worries and obstacles in their business contact, build laws and regulations of information security and data privacy protection, and improve the security and trust system in the application of traffic cloud computing. At the same time, in view of security, stability and network quality of service and other factors which the cloud users are most concerned about, in one side, the software companies, the traditional Internet data center, systems integrators, Internet companies and telecommunication should be upgraded, and to promote the compatibility of technical architecture, data interfaces and 
application mode; in the other side, rules and regulations should be laid down combined with the relevant laws, and make data backup periodically to ensure the security, stable and high-quality of traffic cloud computing implementation.

4.3 Strengthen the service management mechanism, and remove the obstacles of promotion and application

The supervisor department of traffic cloud computing should develop feasible standards, strict procurement control, objective evaluation services and timely management measures combine with platform service provider, to innovate management service, promote traffic information resources integration, and to make sure the promotion of traffic cloud computing service. Meanwhile, for the problems such as purchasing outsourcing practices are not widely accepted, business data do not want to be open in their own building system, a wait-and-see attitude to the cloud computing promotion and application etc., the demonstration based on successful cases can be taken. To organize specialized training, seminars, live demonstrations and online classes for specific user applications of advanced technology, accelerate the popularization and specialization process of traffic cloud computing services by live or online and offline media show.

\section{Acknowledgement}

This paper is sponsored by 2014 Ning Xia Philosophy Social Science Planning Project (14NXBYJ04)

\section{References}

[1] The Chinese government, 2011. "The National Economic and social development Twelfth Five Year Plan of people's Republic of China”.

[2] The CPC Central Committee and State Council, 2010. “The CPC Central Committee and State Council's Decision on accelerating the cultivation and development of strategic emerging industries".

[3] National Development and Reform Commission, Ministry of Industry and Information Technology, 2010. "Notice on cloud computing service innovation and development of pilot and demonstration work".

[4] The CPC Central Committee and State Council, 2011. "Guiding opinions on Accelerating the development of high-tech service industry”.

[5] The Ministry of Commerce, 2013. “Categorized list of government procurement Categories”.

[6] The CPC Central Committee and State Council, 2015. “opinions on promoting the cloud computing innovation and foster-ing the new format of information industry".

[7] The Ministry of Transport, 2015. “Transportation deepen reform opinions”.

[8] Qin, Q. 2013. Research of ITS Based on Cloud Computing. Wu Xian Hu Lian Ke ji (4): 170171.

[9] Shao, Q. Yunpeng, Z. Shaobo, H. 2010. Intelligent Transport Cloud: ITS Based on Cloud Computing. JISUANJIYU XIANDAIHUA (11): 168-171.

[10] Kai, Z. Jinchuan, Y. 2015. Cloud and its application on Intelligent Transportation, MODERN SCIENCE \& TECHNOLOGY OF TELECOMMUNICATIONS (5): 45-51. 